\title{
Doença de Crohn: piora das manifestações clínicas por doenças psíquicas
}

Crohn's disease: worsening of clinical manifestations due to mental illness

Enfermedad de Crohn: empeoramiento de las manifestaciones clínicas por enfermedad mental

Simone Kempf Stachechem ${ }^{1 *}$, Ariany Parreira de Mendonça ${ }^{2}$, Branca Lopes da Silva Guedes ${ }^{3}$, Caroline Kugeratski Carneiro ${ }^{1}$, Gabriela Aparecida Schiefler Gazzoni ${ }^{4}$, Layla Gabriela Alves de Lima ${ }^{5}$, Letícia Lourenço Botelho ${ }^{6}$, Patricia Dupont ${ }^{1}$, Victoria Martins Carrijo ${ }^{7}$, Nestor Augusto Avelino Leite ${ }^{8}$.

\section{RESUMO}

Objetivo: Abordar as principais consequências do estresse na piora das manifestações clínicas da Doença de Crohn (DC), destacando a relevância do cuidado integral ao paciente. Revisão bibliográfica: A DC é crônica e idiopática, com capacidade de afetar qualquer segmento do trato gastrointestinal, caracterizada pelo espessamento globoso resultando em ulcerações e fístulas, podendo evoluir para um quadro mais grave com perfurações e até tumores intestinais. O diagnóstico envolve dados clínicos, achados endoscópicos e radiológicos. A incurabilidade da doença e de sua sintomatologia podem fazer com que os pacientes desenvolvam transtornos psiquiátricos devido ao futuro incerto, causando angústia e sofrimento, essas expectativas sobre a condição refletem diretamente na ansiedade, depressão e qualidade de vida dos portadores da DC. Não tratar os sintomas psíquicos associados à DC repercute em piora significativa do quadro clínico do paciente, afetando consideravelmente a sua qualidade de vida. Considerações finais: Reforça-se a importância da equipe multidisciplinar na abordagem e tratamento de pacientes portadores desta patologia, com o objetivo de garantir a assistência psicológica dos acometidos, melhorando, assim, o prognóstico e a qualidade de vida desses pacientes.

Palavras-chave: Doença de Crohn, Doenças inflamatórias intestinais, Saúde mental, Estresse psicológico.

\begin{abstract}
Objective: Address the main consequences of stress in worsening the clinical manifestations of Crohn's Disease (CD), highlighting the relevance of comprehensive patient care. Bibliographic review: $C D$ is chronic and idiopathic, with the ability to affect any segment of the gastrointestinal tract, characterized by globular thickening resulting in ulcerations and fistulas, which may progress to a more severe condition with perforations and even intestinal tumors. The diagnosis involves clinical data, endoscopic and radiological findings. The incurability of the disease and its symptoms can cause patients to develop psychiatric disorders due to the uncertain future, causing anguish and suffering, these expectations about the condition directly reflect on the anxiety, depression and quality of life of patients with CD. Not treating the psychological symptoms associated with CD results in a significant worsening of the patient's clinical condition, considerably affecting his quality of life. Final considerations: The importance of the multidisciplinary team in the approach and treatment of patients with this pathology is reinforced, with the objective of guaranteeing the psychological assistance of the affected, thus improving the prognosis and quality of life of these patients.
\end{abstract}

Key words: Crohn disease, Inflammatory bowel diseases, Mental health, Psychological stress.

\footnotetext{
1 Universidade do Contestado (UnC), Mafra - SC. *E-mail: simonestachechem@gmail.com

${ }^{2}$ Centro universitário IMEPAC (IMEPAC), Araguari - MG.

${ }^{3}$ Universidade presidente Antônio Carlos (FAME-JF), Juiz de Fora - MG.

${ }^{4}$ Universidade do Vale do Itajaí (UNIVALI), Itajaí - SC.

${ }^{5}$ Faculdade Atenas (UniAtenas), Sete Lagoas - MG.

${ }^{6}$ Centro Universitário de Valença (UNIFAA), Valença - RJ.

7 Instituto Master de Ensino Presidente Antônio Carlos (IMEPAC), Araguari - MG.

${ }^{8}$ Faculdade de Medicina de Campos (FMC), Campos dos Goytacazes - RJ.
} 


\section{RESUMEN}

Objetivo: Abordar las principales consecuencias del estrés en el agravamiento de las manifestaciones clínicas de la Enfermedad de Crohn (EC), destacando la relevancia de la atención integral al paciente. Revisión bibliográfica: La EC es crónica e idiopática, con la capacidad de afectar cualquier segmento del tracto gastrointestinal, caracterizada por un engrosamiento globular que resulta en ulceraciones y fístulas, que pueden progresar a una condición más severa con perforaciones e incluso tumores intestinales. El diagnóstico incluye datos clínicos, hallazgos endoscópicos y radiológicos. La incurabilidad de la enfermedad y sus síntomas pueden provocar que los pacientes desarrollen trastornos psiquiátricos debido al futuro incierto, provocando angustia y sufrimiento, estas expectativas sobre la condición se reflejan directamente en la ansiedad, depresión y calidad de vida de los pacientes con EC. No tratar los síntomas psicológicos asociados a la EC resulta en un empeoramiento significativo de la condición clínica del paciente, afectando considerablemente su calidad de vida. Consideraciones finales: Se refuerza la importancia del equipo multidisciplinario en el abordaje y tratamiento de los pacientes con esta patología, con el objetivo de garantizar la asistencia psicológica del afectado, mejorando así el pronóstico y la calidad de vida de estos pacientes.

Palabras clave: Enfermedad de Crohn, Enfermedades inflamatorias del intestino, Salud mental, Estrés psicológico.

\section{INTRODUÇÃO}

A Doença de Crohn (DC) pertence ao grupo de Doenças Inflamatórias Intestinais (DII) e trata-se de uma patologia inflamatória sistêmica que pode acometer qualquer segmento do trato gastrointestinal. A etiologia dessa doença ainda não foi completamente elucidada, entretanto, os estudos mostram que as principais causas podem ser infecciosas, genéticas, imunológicas, fatores ambientais associados à alimentação, tabagismo, além de fatores relacionados à saúde mental (DIAS PR, et al., 2020; ACCIARI A, et al., 2019).

A DC acomete tanto homens quanto mulheres, porém sua prevalência é mais comum em indivíduos brancos e judeus. Os primeiros sintomas podem aparecer ainda na adolescência, porém observa-se maior incidência entre a segunda e quarta décadas de vida. Estudos epidemiológicos demonstram índices cada vez maiores desta patologia, com aumento de cerca de 15 vezes nas últimas décadas, entretanto a incidência e prevalência da DC variam com a região estudada, sendo mais comum nos países desenvolvidos, acometendo cerca de 200:100.000 habitantes (MARANHÃO DA, et al., 2015; MAURYANE A, et al., 2016).

A multiplicidade de apresentações da DC, semelhanças com outras patologias ou a ocorrência de sintomas discretos ou predominância de manifestações extraintestinais da doença são fatores que dificultam diagnóstico. Dessa forma, é preconizado qeu seja realizada uma análise conjunta de dados clínicos, endoscópicos, histopatológicos e radiológicos (ROMANO S, et al., 2016). A manifestação clínica da doença, por sua vez, abarca diversos sinais e sintomas como fadiga, cólicas intestinais, diarreia crônica e o processo inflamatório característico desta patologia pode provocar complicações como estenoses, abscessos, fístulas e neoplasias (FALCÃO LM e MARTINELLI VF, 2016; MAURYANE A, et al., 2016).

Por se tratar de uma patologia crônica, a DC necessita de tratamento contínuo e prolongado, de forma a manter a qualidade de vida do paciente. A terapêutica clínica inclui tratamento medicamentoso para reduzir a inflamação, diminuir sintomas na fase ativa e manter a remissão (DE SOUZA FG, et al., 2020). O tratamento cirúrgico é indicado nas complicações da doença (obstrução intestinal, hemorragia maciça, megacólon tóxico), na doença refratária ao tratamento clínico e na ocorrência de displasia ou câncer. É recomendável, ainda, não fumar, evitar uso de Antiinflamatórios Não Esteroides (AINE's) e acompanhamento nutricional para reposição de nutrientes e uso de probióticos (FEUERSTEIN JD e CHEIFETZ AS, 2017).

Em decorrência da incurabilidade da doença e de sua sintomatologia, é comum que seus portadores desenvolvam transtornos mentais, como ansiedade e depressão que, em muitos casos, influenciam no agravamento de alguns sintomas (ACCIARI A, et al., 2019; SAJADINEJAD MS, et al., 2012). O estresse e os outros sintomas psíquicos apresentados pelo paciente, provocam, frequentemente, a piora da condição clínica da DC, resumindo a realidade desses indivíduos à uma decadência cíclica: a doença leva ao desenvolvimento de transtornos psíquicos e estes transtornos, por sua vez, agravam o quadro clínico da própria doença (REIGADA L, et al., 2015). 
Sabe-se que os sintomas podem impactar a vida social dos indivíduos, tornando de extrema importância a investigação de determinantes sociais, fisiológicos e psicológicos possivelmente envolvidos no agravamento da doença. Além disso, a busca pela garantia de uma boa qualidade de vida para os portadores da doença é extremamente importante, abordando-os de forma ampla e com a implementação de medidas como a psicoterapia, que tem um efeito positivo na dimensão da doença, favorece o bem-estar psicológico e a criação de estratégias de enfrentamento da doença e de alívio do estresse (ROMANO S, et al., 2016).

A partir do exposto, o objetivo deste trabalho foi abordar as principais consequências do estresse na piora das manifestações clínicas da DC, destacando a relevância do cuidado integral ao paciente.

\section{REVISÃO BIBLIOGRÁFICA}

As DII compreendem doenças crônicas que inflamam o intestino em intensidades e regiões variadas. As principais formas das DII são a DC e a Retocolite Ulcerativa (RU) ou colite ulcerativa. Tais doenças apresentam diversas manifestações clínicas que podem afetar qualquer parte do trato gastrointestinal (TGI), entretanto, acometem principalmente a porção final do intestino delgado e cólon (ACCIARI A, et al., 2019; MAURYANE A, et al., 2016; MARANHÃO DA, et al., 2015).

Assim, a DC é uma patologia crônica e idiopática e suas lesões comprometem todas as camadas, desde a mucosa até a serosa e sua ocorrência está presente em todo o mundo, entretanto sua distribuição, tanto em incidência como em prevalência, não é homogênea (CARDOZO WS e SOBRADO CW, 2015). Embora os fatores que contribuem para o desenvolvimento dessa patologia ainda não tenham sido totalmente elucidados, é considerada uma doença de populações desenvolvidas, com o estilo de vida ocidental contribuindo fortemente para seu desenvolvimento e taxas de incidência e prevalência elevadas em regiões como a América do Norte e Norte Europeu (BURISH J e MUNKHOLM P, 2013; HA F e KHALIL H, 2015).

O Brasil é considerado um país de baixa incidência e prevalência de DC, embora note-se um claro crescimento no volume de atendimentos e internações de pacientes com esta doença. Poucos foram os estudos epidemiológicos nacionais sobre o tema, possivelmente devido à inexistência, até o momento, de sistemas integrados de notificação de diagnóstico das DII. Entretanto, um número cada vez maior de estudos regionais vem sendo realizados, o que pode fornecer melhores dados sobre a prevalência e incidência da DC no país (GASPARINI RG, 2018).

Sabe-se que a DC tem caráter multifatorial, envolvendo fatores imunológicos, genéticos, bem como fatores de risco que desencadeiam o seu surgimento, entre eles a alimentação, tabagismo, utilização prolongada de AINE's, infecções intestinais e o uso de contraceptivos orais (TOMAZONI EI e BENVEGNÚ DM, 2017; PAPACOSTA NG, et al., 2017). Quanto aos fatores imunológicos, alguns autores relacionam a etiopatogenia da doença a uma resposta imunológica anormal à microbiota bacteriana intestinal, que gera alterações na função de barreira da mucosa. Assim, alterações nessa barreira, com consequente alteração em sua permeabilidade, resulta em um aumento da passagem de antígenos, o que resultaria em resposta imune exacerbada e em inflamação crônica (MARANHÃO DA, et al. 2015).

A fisiopatologia inicial da DC é caracterizada pelo espessamento globoso resultando em ulcerações e fístulas que podem penetrar profundamente e criando trajetos fistulosos envolvendo-se com outras alças do intestino. À medida que evolui para um quadro mais grave, podem surgir perfurações, obstruções e até mesmo tumores intestinais. Com o desenvolvimento da doença, o estado nutricional dos pacientes pode ser afetado em virtude da redução na ingestão alimentar e perda de nutrientes causada por sintomas gastrointestinais como diarreia, sangue nas fezes, oclusão intestinal, abdome agudo e má absorção (TOMAZONI El e BENVEGNÚ DM, 2017; GUIMARÃES MC, et al., 2020; SANTOS LA, et al., 2015).

A DC pode evoluir de forma crônica e progressiva ou com crises intermitentes, alternadas com fases de remissão de duração variável, podendo o paciente apresentar-se assintomático em uma fase inicial da doença, porém com manifestações clínicas intensas com o acometimento de extensões maiores do trato digestivo (DIAS PR, et al., 2020; GASPARINI RG, 2018). A patologia se apresenta em três padrões principais: doença no íleo e ceco, doença restrita ao intestino delgado e doença restrita ao cólon e essa localização está intimamente relacionada à manifestação clínica da doença (MARANHÃO DA, et al., 2015). 
Ainda assim, o quadro clínico da DC é heterogêneo e caracteriza-se por sintomas gastrointestinais, extraintestinais ou a combinação de ambos. Tipicamente incluem dor abdominal, diarreia (muitas vezes com muco e sangue), tenesmo, emagrecimento, podendo ainda apresentar febre, anorexia e mal-estar geral. Pode evoluir para o surgimento de abscessos, fístulas (muitas vezes perianais) e obstrução intestinal por estenoses (CARDOZO WS e SOBRADO CW, 2015). Já as manifestações extraintestinais podem evoluir com comprometimento articular, dermatológico, hepático, oftalmológico, entre outros (FERREIRA IF, et al., 2020; GASPARINI RG, 2018).

A confirmação diagnóstica da DC baseia-se na anamnese e exame físico, exames laboratoriais, avaliação endoscópica, radiológica e histológica (HA F e KHALIL H, 2015). Os procedimentos endoscópicos permitem que haja visualização direta, acesso ao lúmen intestinal e, durante o exame, podem ser encontradas estenoses luminais, fístulas, erosões e úlceras aftosas. Já as técnicas de imagem transversal, incluindo tomografia computadorizada, ressonância magnética e ultrassonografia se fazem importantes na investigação e acompanhamento da DC (FEUERSTEIN JD e CHEIFETZ AS, 2017; VEAUTHIER B e HORNECKER JR, 2018; GUIMARÃES MC, et al., 2020).

O tratamento clínico da DC utiliza diferentes fármacos como os aminossalicilatos e corticosteróides sendo eficazes em induzir a remissão da doença; os antibióticos nos casos de perfuração, hemorragia maciça, megacólon tóxico e doença ativa sem resposta ao tratamento e os imunossupressores, que são utilizados no tratamento da forma grave da DC, sendo úteis em pacientes em que as doses de corticoides não podem ser reduzidas ou descontinuadas. O tratamento cirúrgico, por sua vez, está relacionado ao tempo da doença e falhas no tratamento clínico, sendo indicado em casos de obstrução intestinal total ou parcial, fístulas, abcessos e massas inflamatórias, hemorragias, perfurações e neoplasia maligna (ROMANO S, et al., 2016).

O tempo do tratamento pode variar de paciente para paciente, pois a tolerância à medicação difere entre os organismos, influenciando no tempo de resposta dos fármacos, além do local e a gravidade da doença. Quanto maior o tempo entre o diagnóstico e o início do tratamento, maiores são as chances de o local afetado pela patologia apresentar graves e irreversíveis lesões, o que dificulta e prolonga o tratamento em si (ACCIARI A, et al., 2019; APARICIO AS, 2013).

Assim, por se tratar de uma desordem crônica, com episódios de crises e remissões, de início muitas vezes precoce, de curso imprevisível, com necessidade de tratamento a longo prazo, seus portadores passam longos períodos em tratamento e com necessidade de cuidados especiais. Dessa forma, a abordagem terapêutica da doença, além da terapia nutricional, baseia-se na indução e manutenção da remissão, controle dos sintomas, tratamento cirúrgico quando necessário e melhoria da qualidade de vida (FALCÃO LM e MARTINELLI VF, 2016).

Diante do diagnóstico confirmado, os pacientes passam a conviver com a sintomatologia da DC e, consequentemente, com o alto grau de estresse causado pela doença, ficando sujeitos a desenvolver patologias de saúde mental como a ansiedade e depressão. O oposto também pode ocorrer, uma vez que transtornos emocionais tendem a desencadear ou agravar as crises dessa doença inflamatória (ASSOCIAÇÃO BRASILEIRA DE COLITE ULCERATIVA E DOENÇA DE CROHN, 2020; REIGADA L, et al., 2015). Além disso, esses pacientes precisam ter novas responsabilidades a partir do diagnóstico da doença, como o uso de medicações controladas, bem como conhecer a doença e lidar com incômodos físicos e sociais, fatores esses que podem modificar muito a sua rotina de vida, representando para eles um futuro incerto, causando angústia e sofrimento (LEITE VM, et al., 2019).

Já no século XVIII, Sigmund Freud destacou o papel do sofrimento psicológico no desencadeamento de alterações orgânicas como diarreia, constipação e dor abdominal. Sabe-se que, inicialmente, as DII foram consideradas doenças psicossomáticas, e embora atualmente a compreensão da causalidade seja multifatorial, todo o trato gastrointestinal é afetado por sintomas somáticos com possível influência de aspectos emocionais. Desse modo, não só as percepções dos pacientes sobre a doença podem aumentar a tensão mental e emocional dos indivíduos, como também expectativas sobre a condição refletem diretamente na ansiedade, depressão e qualidade de vida dos portadores da DC (LIMA FD, et al., 2012; SAJADINEJAD MS, et al., 2012). 
Alguns estudos já evidenciaram que os indivíduos portadores de DIl são mais vulneráveis a desenvolver distúrbios psicológicos. Uma vez que as pessoas geram sentimentos negativos direcionados à sua patologia, esses gradativamente se convertem em um estado psíquico negativo não colaborante para o tratamento contínuo e crônico do problema de saúde em questão. Logo, observa-se alta correlação entre aspectos emocionais e DIl, e sugerem que algumas desordens psicológicas podem ser uma consequência da DC, enquanto que o grau de angústia psicológica e as perturbações estão relacionados à gravidade desta doença (ZHANG M, et al. 2016).

Lima FD, et al. (2012) demonstraram que $58 \%$ dos pacientes com DC apresentam alta incidência de oscilação do humor, com predomínio do distúrbio para o gênero feminino. A depressão já foi relatada como fator de risco para pior prognóstico da DII, bem como de menor resposta à terapia farmacológica. Causa, ainda, prejuízo nas relações interpessoais e promove absenteísmo e afastamento das atividades laborais. Destaca-se na literatura a ocorrência da exacerbação de DC nos períodos de ansiedade ou depressão e citase a rede psiconeuroendócrina como moduladora da dor e da inflamação para explicar tal fato. Pesquisadores evidenciam, ainda, associação significativa entre o início do tratamento farmacológico da depressão e o alívio das manifestações gastrointestinais (FALCÃO LM e MARTINELLI VF, 2016).

Uma pesquisa realizada no Japão com 1078 pessoas, sendo dessas 303 com DC, observou que 75\% dos pacientes acreditavam que o estresse psicológico desencadeado pela doença causava exacerbação do quadro clínico. O estresse a que esses pacientes são submetidos pode gerar uma quebra da homeostase natural, podendo iniciar, amplificar ou mesmo manter a inflamação da mucosa intestinal. Esse fenômeno é mediado pelo Sistema Nervoso Autônomo (SNA) por meio de um efeito na estimulação do eixo HipotálamoPituitária-Adrenal (HPA) interagindo diretamente com as células imunes (ARAKI M, et al., 2020; FALCÃO LM e MARTINELLI VF, 2016).

A ansiedade e estresse estão associados, também, à modificação na função de bloqueio da mucosa. $\mathrm{Na}$ DC, ocorre um aumento da permeabilidade na barreira intestinal, em razão da amplificação da passagem de antígenos e agentes pró inflamatórios, como resultado, ocorre perda da tolerância à flora comensal e inflamação crônica. Entretanto, é possível que o estresse induza essas alterações intestinais através do eixo hipotálamo-adrenal, por meio da mediação de hormônios, como o hormônio adrenocorticotrófico, que foi encontrado no trato intestinal dos pacientes com DC (GUIMARÃES MC, et al., 2020).

Um importante ponto a ser discutido é a relação da ansiedade com a rigidez aórtica na DC. A ansiedade executa ações na ativação do Sistema Nervoso Simpático (SNS) e na supressão do Nervo Vago, o qual afeta a saúde mental e, dentre suas funções, estão o controle da pressão arterial e dos batimentos cardíacos. Dessa forma, a alteração do equilíbrio do sistema simpático/parassimpático, ou seja, na presença de ansiedade e depressão prolongadas, desempenha um papel na patogênese e progressão das DII, podendo levar a um aumento da pressão arterial, da rigidez arterial por meio da ativação do sistema simpático e com isso a piora inflamatória (ZANOLLI L, et al., 2020).

Os distúrbios psicológicos e menor qualidade de vida podem acometer cerca de $50 \%$ dos pacientes com DC, sendo que a gravidade da doença pode associar-se ao grau de sofrimento psíquico. Além disso, a tensão emocional pode influenciar a evolução da DC e, dessa forma, tem-se a importância de um adequado acompanhamento psicológico, e eventualmente acompanhamento psiquiátrico, para receber suporte emocional, desenvolver recursos para lidar os estressores relacionados à doença, reduzir tensão, a ansiedade, a depressão e o estresse (TOMAZONI EI BENVEGNÚ DM, 2018).

Diante disso, ao não tratar os sintomas psíquicos associados à DC reconhece-se uma piora significativa do quadro clínico do paciente, independente da gravidade, afetando consideravelmente a sua qualidade de vida. Por isso, reforça-se a importância da equipe multidisciplinar e intersetorial na abordagem e tratamento de pacientes portadores dessa doença, ampliando recursos técnicos, estruturais e financeiros para o tratamento dos transtornos psiquiátricos a fim que não agravar de forma significativa a história natural da doença (DOS SANTOS, et al., 2020; TOMAZONI EI BENVEGNÚ DM, 2018).

Outro fator de melhora que pode ser adicionado ao tratamento dos transtornos psíquicos durante a condição de DC é a prática de atividades físicas, sendo essa um meio eficaz já comprovado de 
acompanhamento do tratamento da ansiedade e depressão. Estudos relatam que os praticantes de exercícios físicos, sendo acompanhados por um profissional da área, ou ao ar livre de forma espontânea, reduzem a frequência dessas comorbidades. Entretanto, sugere que mais estudos são necessários para fornecer dados ainda mais precisos sobre essa associação (DOS SANTOS, et al., 2020).

\section{CONSIDERAÇÕES FINAIS}

A partir da literatura analisada observou-se que os pacientes portadores de DC podem desenvolver transtornos psiquiátricos frente à difícil convivência com a cronicidade da doença e sintomatologia que exigem longos períodos em tratamento e a necessidade de cuidados especiais. Esses transtornos, por sua vez, podem agravar o quadro clínico da doença, levando à uma decadência cíclica. Logo, faz-se necessário mais estudos sobre a patologia a fim de aprimorar a assistência terapêutica a esses indivíduos e reforça-se a importância da assistência psicológica dos acometidos de forma a, reduzir os transtornos mentais e suas consequências, melhorando, assim, o prognóstico e a qualidade de vida desses pacientes.

\section{REFERÊNCIAS}

1. ASSOCIAÇÃO BRASILEIRA DE COLITE ULCERATIVA E DOENÇA DE CROHN (ABCD). Emoções influenciam na DII. 2020. Disponível em: https://www.abcd.org.br/blog/noticias/emocoes-influenciam-na-dii/. Acessado em: 14 de maio de 2021.

2. ACCIARI A, et al. Relationship among psychological well-being, resilience and coping with social and clinical features in Crohn's disease patients. Arquivos de Gastroenterologia, 2019; 56(2): 131-140.

3. ARAKI M, et al. Psychologic stress and disease activity in patients with inflammatory bowel disease: A multicenter cross-sectional study. PLoS One, 2020; 15(5): 1-12.

4. APARICIO AS. Doença Inflamatória Intestinal: Manutenção Clínica e Novos Tratamentos. Dissertação (Mestrado em Medicina) - Universidade da Beira Interior, 2013; 30 p.

5. BURISH J, MUNKHOLM P. Inflammatory bowel disease epidemiology. Current opinion in gastroenterology, 2013; 29: 357-362.

6. CARDOZO WS, SOBRADO CW. Doença Inflamatória Intestinal. 2ed. Barueri-SP: Manole, 2015; 772p.

7. DE SOUZA FG, et al. Adesão ao tratamento farmacológico em pacientes com doenças inflamatórias intestinais: uma revisão integrativa da literatura. Revista Eletrônica Acervo Saúde, 2020; 13(2): 1-11.

8. DIAS PR, et al. A relação do microbioma intestinal e o sistema imune no desenvolvimento da doença de Crohn. Revista Eletrônica Acervo Científico, 2020; 17: e5618.

9. DOS SANTOS CM, et al. Análise comparativa da prevalência de ansiedade e depressão entre indivíduos portadores e não portadores de doença inflamatória intestinal. Journal of Coloproctology, 2020; 40(4): 339-344..

10. FALCÂO LM. MARTINELLI VF. Associação de doença inflamatória intestinal com ansiedade e depressão: avaliação dos fatores de risco. Gastroenterologia Endoscopia Digestiva (GED), 2016; 35(2): 52-58.

11. FERREIRA IF, et al. Manifestações cutâneas na doença de Crohn: diagnóstico e prognóstico. Revista Eletrônica Acervo Científico, 2020; 13: e4690.

12. FEUERSTEIN JD, CHEIFETZ AS. Crohn disease: epidemiology, diagnosis, and management. In: Mayo Clinic Proceedings. Elsevier, 2017; p1088-1103.

13. GASPARINI RG. Incidência e Prevalência de Doenças Inflamatórias Intestinais no Estado de São Paulo - Brasil. Tese (Doutorado em Bases Gerais da Cirurgia) - Faculdade de Medicina, Universidade Estadual Paulista "Júlio de Mesquita Filho", Botucatu, 2018; 91p.

14. GUIMARÃES MC, et al. Doença De Crohn: Um Estudo De Caso. Humanidades e Tecnologia (FINOM), 2020; 23(1): 343-361.

15. HA F, KHALIL H. Crohn's disease: a clinical update. Therapeutic advances in gastroenterology, 2015; 8(6): $352-359$.

16. LEITE VM, et al. Impacto dos aspectos psicológicos em portadores de doença inflamatória intestinal. Brazilian Journal of Health Review, 2019; 3(2): 1363-1367.

17. LIMA FD, et al. Oscilação do humor em pacientes com doença de Crohn: incidência e fatores associados. Revista da Associação Médica Brasileira, 2012; 58(4): 481-488.

18. MAURYANE A, et al. Qualidade de vida de pacientes com doença de Crohn. Enfermaria Global, 2017; 16(3): 321368.

19. MARANHÃO DA, et al. Características e diagnóstico diferencial das doenças inflamatórias intestinais. Jornal Brasileiro de Medicina, 2015; 103(1): 9-15.

20. PAPACOSTA NG, et al. Doença de Crohn: um artigo de revisão. Revista de Patologia do Tocantins, 2017; 4(2): 2535.

21. REIGADA L, et al. Anxiety Symptoms and Disease Severity in Children and Adolescents with Crohn Disease. Journal of Pediatric Gastroenterology and Nutrition, 2015; 60, 6: 1-6.

22. ROMANO S, et al. Doença de Crohn, diagnóstico e tratamento. Atas de Ciências da Saúde, 2016; 4(4): 31-50. 
23. SAJADINEJAD MS, et al. Psychological issues in inflammatory bowel disease: an overview. Gastroenterology Research and Practice, 2012; 2012: 1-11.

24. SANTOS LA, et. al. Terapia nutricional nas doenças inflamatórias intestinais: artigo de revisão. Nutrire, 2015; 40(3): 383-396.

25. TOMAZONI EI, BENVEGNÚ, DM. Sintomas de ansiedade e depressão, e a qualidade de vida de pacientes com doença de Crohn. Arquivos de Gastroenterologia, 2018; 55(2): 148-153.

26. VEAUTHIER B, HORNECKER JR. Crohn's disease: Diagnosis and management. American family physician, 2018; 98(11): 661-669.

27. ZANOLLI L, et al. Anxiety, depression, chronic inflammation and aortic stiffness in Crohn's disease: the brain--gut-vascular axis. Journal of Hypertension, 2020; 38(10): 1-10.

28. ZHANG M, et al. Illness perceptions and stress: mediators between disease severity and psychological well-being and quality of life among patients with Crohn's disease. Patient Preference and Adherence, 2016; 23(10): 2387-2396. 